\title{
The 8-Year Management of an Older Breast Cancer Patient by Non-surgical Primary Therapies and Minimized Surgery: A Case Report
}

\author{
Jin Kyung $\mathrm{An}^{1}$, Jeong Joo Woo ${ }^{1}$, Hee Yong Kwak ${ }^{2}$ \\ ${ }^{1}$ Department of Radiology, Nowon Eulji Medical Center, Eulji University, Seoul, Korea \\ ${ }^{2}$ Department of Surgery, Nowon Eulji Medical Center, Eulji University, Seoul, Korea
}

\author{
Corresponding Author: \\ Jin Kyung An, MD \\ Department of Radiology, Nowon Eulji \\ Medical Center, Eulji Univeristy, 68 \\ Hangeulbiseok-ro, Nowon-gu, Seoul \\ 01830, Korea \\ E-mail: jkan0831@eulji.ac.kr \\ ORCID: \\ https://orcid.org/0000-0003-1086-426X
}

Received: April 23, 2020

Revised: July 3, 2020

Accepted: July 3, 2020

\begin{abstract}
A 74-year-old woman presented with a palpable lesion in her right breast. At the time of her visit, she was taking medications for diabetes, hypertension, tremors, tinnitus, and lumbago. She was also caring for her husband, who had dementia. Imaging studies revealed another lesion in addition to the palpable mass. A core biopsy of the palpable mass confirmed invasive ductal carcinoma. Surgery was recommended to remove both masses. However, the patient refused the operation due to her comorbidities and her husband's nursing needs. A modified treatment process, including non-surgical primary therapies, minimized surgery, and close follow-up, for 8 years proved successful. This report focuses on the points to consider in the treatment and management of older patients with breast cancer.
\end{abstract}

Key Words: Breast neoplasms, Aged, Therapeutics, Disease management

\section{INTRODUCTION}

Breast cancer is one of the most common female malignancies with an increasing incidence not only in Western but also in Asian countries. In particular, the rate of older patients with breast cancer is rising with the increase in the number of older people. ${ }^{1-4)} \mathrm{Al}-$ though there are no set standards for aged patients, the tumor biological characteristics differ between patients aged over 70 or 80 years compared to those in younger patients. Lodi et al..$^{5)}$ reported larger tumor sizes, more frequent positive lymph nodes and distant metastases, and higher breast cancer-specific mortality in patients aged over 80 years than in patients aged 70 to 79 years. Jeon et al. ${ }^{6}$ reported larger tumor sizes and higher pathologic stages in patients aged over 75 years than in patients younger than 75 years.

While various guidelines have been recommended by countries and organizations for breast cancer diagnosis and treatment, it is difficult to apply uniform treatments to older patients with breast cancer who are more susceptible to comorbidities and have less ability to tolerate and adhere to treatment than younger people.
Thus, modified treatment is required in these cases. This case report describes an older patient with breast cancer who was maintained for 8 years with non-surgical primary treatments and minimized surgery. We also present some points to consider regarding older patients with breast cancer as the aging population grows.

\section{CASE REPORT}

In 2011, a 74-year-old woman visited our hospital with a palpable lesion in her right breast. Physical examination revealed a hard, movable, and non-tender mass. The patient had hypertension and diabetes both diagnosed 10 years ago. Laboratory studies revealed high hemoglobin Alc, serum glucose, and urine micro-albumin levels. She had diabetic retinopathy and had undergone pseudophakia surgery. She had symptoms of resting and postural regular tremors, especially in her finger, as well as intermittent tinnitus in both ears. She was diagnosed with lumbar stenosis and had difficulty in movement due to lumbago. She was taking various medications for her comorbidities. Although she had been recommend- 
ed to undergo further breast studies a year earlier, she could not spare time to be examined because she was taking care of her husband, who had dementia.

The mammogram showed an irregular hyperdense mass in the right upper central deep breast (Fig. 1A, 1B). Ultrasound (US) of the palpable lesion showed an approximately $2.7-\mathrm{cm}$ irregular hypoechoic mass in the right upper central breast located $3-\mathrm{cm}$ from the right nipple (Fig. 1C). An additional $0.8-\mathrm{cm}$ irregular hypoechoic mass was found on the right upper outer breast, $2.5-\mathrm{cm}$ from the large palpable mass (Fig. 1D). Multifocal breast malignancy was strongly suspected. No suspicious lesions were identified in the left breast or either axilla. A US-guided core biopsy of the palpable mass in the right upper central breast confirmed invasive ductal carcinoma. The modified Black nuclear and histologic grade was 2 and immunohistochemical analyses showed estrogen receptor (ER), progesterone receptor (PR), human epidermal growth factor receptor 2 (HER2), and tumor suppressor p53-positivity, as well as moderate $\mathrm{Ki}-67$ protein.

Surgery was recommended based on the imaging findings and pathology results. Since the two masses were located $2.5-\mathrm{cm}$ apart, the range of surgery was wider than if only one mass was removed. Even when considering the separate removal of the two masses, one of the lesions required additional preoperative procedures for breast localization due to its small size. Therefore, regardless of which method was selected, general anesthesia was required for surgery. However, the patient refused any operation due to her comorbidities and her need to care for her husband. Thus, endocrine and radiation therapy were chosen as treatment alternatives. F-18 fluorodeoxyglucose (FDG) PET-CT was performed to confirm that there was no distant metastasis and bone mineral densitometry was performed before medication was administered. She un- derwent radiation therapy with a total of 5,000 cGy (25 fractions in 5 weeks) to the whole breast followed by $1,600 \mathrm{cGy}$ ( 8 fractions over 2 weeks) for the breast mass. She was prescribed a selective estrogen receptor modulator, Fareston (Orion Corporation, Espoo, Finland), along with Dicamax (DalimBiotech, Seoul, Korea) and Bonviva (Roche, Basel, Switzerland) to manage osteoporosis.

At 3- and 9-month follow-ups after treatment initiation, the right upper central palpable mass had gradually decreased in size and was not observed at all in the US conducted one and a half years later (Fig. 2). The right upper outer small lesion had slightly decreased at the 9-month follow-up after treatment initiation and was measured at the smallest size 2 years 4 months later (Fig. 3A). The patient continued to take medication, accompanied by breast US and physical examinations. At the 4 years 4 months follow-up, the right upper central mass remained invisible; however, the right upper outer lesion, which had been somewhat stable, began to increase in size (Fig. 3B). On the subsequent follow-ups, the lesion size increased continuously (Fig. 3C, 3D). We recommended surgery again due to the clear growing trend of the mass. However, the patient was very reluctant to undergo general anesthesia. Therefore, we instead performed an excision under local anesthesia and finally removed the remaining mass 5 years 7 months since the first diagnosis of breast malignancy. The mass was confirmed as invasive ductal carcinoma, measuring $2.0 \times 1.0 \times 1.0 \mathrm{~cm}^{3}$. Immunohistochemistry showed ER and PR positivity, and borderline HER2 positivity. After surgery, the patient was prescribed an aromatase inhibitor, Femara (Novartis, Basel, Switzerland), as well as Dicamax and Fosamax (Merck Sharp \& Dohme Corp., Kenilworth, NJ, USA) to manage osteoporosis. A follow-up study conducted in July 2019, 2 years after the surgery and 8 years after the first diagnosis, showed no signs of recurrence or metastasis.

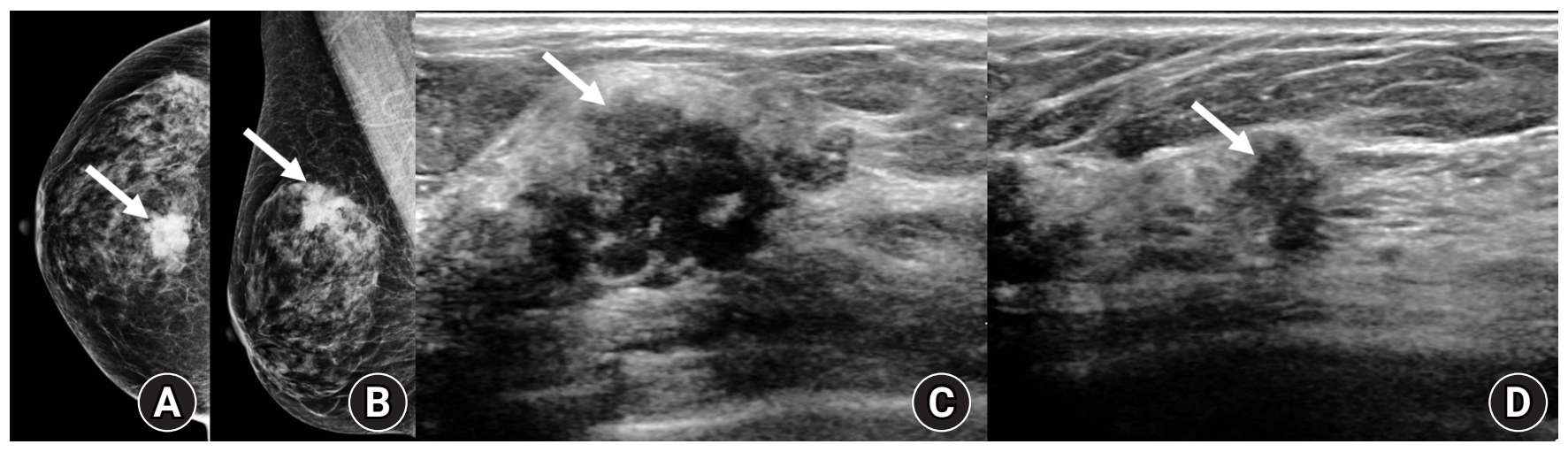

Fig. 1. Initial mammogram and ultrasonography of the patient. (A, B) Right craniocaudal and mediolateral oblique mammogram showing an irregular hyperdense mass in the right upper central deep breast with a spiculated margin. (C) On ultrasound, the palpable lesion shows an approximately $2.7-\mathrm{cm}$ irregular hypoechoic mass with angular margin and microcalcifications in the right upper central breast. (D) An additional small irregular hypoechoic mass was found in her right upper outer breast, with an angular margin and vertical orientation. 


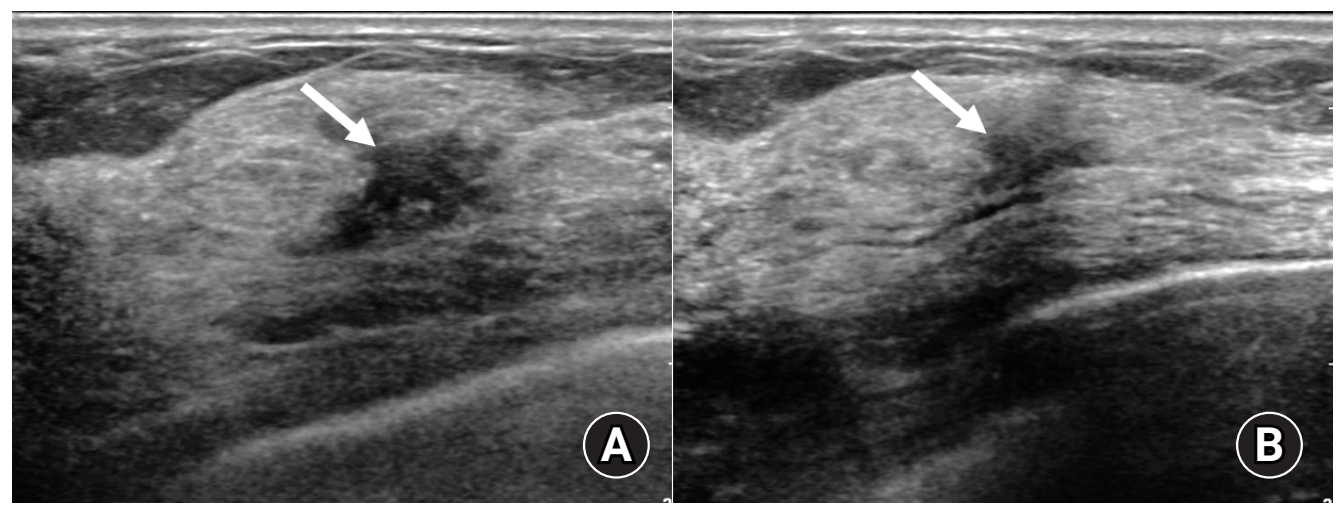

Fig. 2. Follow-up ultrasound of the right upper central mass. At the 3-month follow-up (A) and 9-month follow-up (B) after treatment initiation, the right upper central mass had gradually decreased in size. The mass completely had disappeared a year and a half later and the exact location of the mass was not visible on ultrasound.

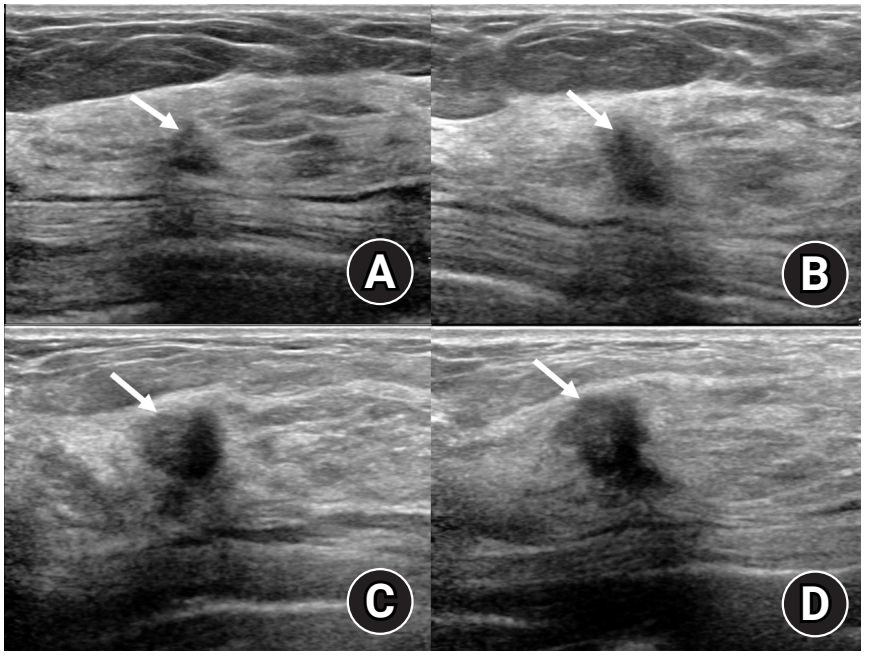

Fig. 3. Follow-up ultrasound of the right upper outer mass. (A) The small lesion in the right upper outer breast began to shrink after 9 months of treatment and was measured at the smallest size 2 years 4 months later. (B) At the 4 years 4 months follow-up, the right upper outer lesion, which had been somewhat stable, showed suspicious enlargement. (C) Ultrasound conducted 5 years 4 months later showing a prominent increase in lesion size. (D) Four months later, the lesion has further increased in size beyond its initial measurements.

This report was approved by the Institutional Review Board of our hospital, with informed consent from the patient waived.

\section{DISCUSSION}

Breast cancer is one of the most common female cancers worldwide, accounting for $24.2 \%$ of new cases in 2018. ${ }^{1)}$ Statistics compiled in Korea in 2017 also showed breast cancer to be the most common type of cancer in women, representing $20.3 \%$ of new fe- male cancers. ${ }^{2)}$ The rate of breast cancer has been rising constantly since 1999 when statistics first started to be compiled in Korea. As a result, the proportion of older patients with breast cancer has also increased, with the incidence of breast cancer at age 70 years or older increasing from $5.34 \%$ (444/5,708 cases) in 2003 to $9.87 \%$ $(2,148 / 21,747$ cases $)$ in $2016.3,4)$

Many countries have developed their own guidelines for breast cancer diagnosis and treatment. The Korean Society for Breast Cancer announced its 8th medical recommendation in 2019. The policy for older patients is that sentinel lymph node biopsy or axillary lymph node dissection can be selectively conducted if the information on the axillary lymph node does not affect the patient's treatment decision. Postoperative radiation therapy is not conducted in patients aged over 70 years with tumors measuring less than 3 -cm, negative axillary lymph nodes, positive hormone receptors, and clear resection margins, taking into account the comorbidities and life expectancy; and endocrine therapy alone is also possible. For patients aged 70 years and older, chemotherapy is individually determined based on the patient's comorbidities and general condition. ${ }^{7)}$ The National Comprehensive Cancer Network (NCCN) separately provides guidelines for "older adult oncology" in which patients with high risks of dying or suffering from cancer to receive palliative care. In treatable conditions, patient ability to withstand cancer treatment or comprehensive geriatric assessment should be evaluated in advance. ${ }^{8)}$

In our case, the treatment choices included breast conserving surgery with or without radiation therapy or mastectomy, sentinel lymph node biopsy with or without axillary lymph node dissection, endocrine therapy based on ER/PR positivity, and/or chemotherapy.

Endocrine therapy is a useful non-surgical treatment for ER-positive older patients. A study in women aged over 75 years who took 
hormonal therapy without surgery despite being surgical candidates, reported that the reasons for primary endocrine therapy included older age, increased comorbidities, and patient choice. ${ }^{9)}$ The International Society of Geriatric Oncology (SIOG) and European Society of Breast Cancer Specialist (EUSOMA) updated their recommendations in 2012 to support the use of primary endocrine therapy for patients with hormone receptor-positive tumors with short life expectancy or who are unfit for or refuse surgery. ${ }^{10)}$ Endocrine therapy can significantly reduce the mass size although at a slower rate than that with chemotherapy. Primary endocrine therapy can control tumor growth for approximately 18 to 24 months. The time to onset of response may take several months or longer. ${ }^{11)}$

Radiation therapy is usually offered to patients with tumors larger than 5-cm, locally advanced tumors, close surgical margins near the tumors, and more than four positive axillary lymph nodes to lower local recurrence. Poleszczuk et al. ${ }^{12)}$ reported that preoperative radiotherapy for early-stage breast cancer may improve disease-free survival without decreasing overall survival. They suggested preoperative radiotherapy followed by preoperative endocrine therapy and lumpectomy. The NCCN Guidelines recommend that considerations for older patients undergoing radiation therapy depend on the anatomic site being radiated and the dose/fractionation chosen. ${ }^{8)}$

In this case, radiotherapy and endocrine therapy were administered simultaneously and a decrease in one of the mass was confirmed at 3-month follow-up. Thus, we could not determine which treatment had a greater effect. However, since radiation therapy was implemented in the early stages of treatment and endocrine therapy continued, we believe that endocrine therapy played a major role in stabilizing the remaining mass for approximately 5 years before it increased in size. The ultimate treatment for breast cancer is surgery. This patient underwent surgery for the mass under local anesthesia due to her reluctance regarding general anesthesia.

In conclusion, although surgery is the ultimate treatment for breast cancer, a modified treatment process could be considered for older patients with physical limitations and complex comorbidities. The patient's choice or rejection of surgery are also important reasons for considering alternative treatments. Hormonal or radiation therapy could be an important primary treatment in those cases. If the lesions remain and the patient is cooperative, close follow-ups can be implemented to detect lesion progression. If the lesion is small enough and general anesthesia is risky for the patient, surgery with local anesthesia may be a possible alternative.

\section{ACKNOWLEDGMENTS}

\section{CONFLICT OF INTEREST}

The researchers claim no conflicts of interest.

\section{AUTHOR CONTRIBUTIONS}

Conceptualization, JKA; Writing-original draft, JKA; Writing-review \& editing, JJW, HYK.

\section{REFERENCES}

1. Bray F, Ferlay J, Soerjomataram I, Siegel RL, Torre LA, Jemal A. Global cancer statistics 2018: GLOBOCAN estimates of incidence and mortality worldwide for 36 cancers in 185 countries. CA Cancer J Clin 2018;68:394-424.

2. National Cancer Information Center. Cancer statistics in 2017 [Internet]. Goyang: National Cancer Information Center; 2017 [cited 2020 Jul 13]. Available from: https://www.cancer.go.kr/ lay1/S1T639C641/contents.do.

3. Korea Central Cancer Registry. Annual report of cancer statistics in Korea in 2003. Goyang: Central Cancer Registry; 2008. p. 389.

4. Korea Central Cancer Registry. Annual report of cancer statistics in Korea in 2016. Goyang: Korea Central Cancer Registry; 2019. p. 54-5.

5. Lodi M, Scheer L, Reix N, Heitz D, Carin AJ, Thiebaut N, et al. Breast cancer in elderly women and altered clinico-pathological characteristics: a systematic review. Breast Cancer Res Treat 2017; 166:657-68.

6. Jeon YW, You SH, Lee JE, Youn HJ, Lim W, Han JH, et al. Optimal treatment of breast cancer in women older than 75 years: a Korea Breast Cancer Registry analysis. Breast Cancer Res Treat 2019;178:693-701.

7. Korean Breast Cancer Society. The 8th Korean clinical practice guideline for breast cancer. Seoul: Korean Breast Cancer Society; 2019. p. 67-84.

8. National Comprehensive Cancer Network. NCCC Guidelines for specific populations: older adult oncology [Internet]. Plymouth Meeting, PA: National Comprehensive Cancer Network; c2020 [cited 2020 Jul 13]. Available from: https:// www.nccn. org/professionals/physician_gls/default.aspx.

9. Wink CJ, Woensdregt K, Nieuwenhuijzen GA, van der Sangen MJ, Hutschemaekers S, Roukema JA, et al. Hormone treatment without surgery for patients aged 75 years or older with operable breast cancer. Ann Surg Oncol 2012;19:1185-91.

10. Biganzoli L, Wildiers H, Oakman C, Marotti L, Loibl S, Kunkler I, et al. Management of elderly patients with breast cancer: updated recommendations of the International Society of Geriatric Oncology (SIOG) and European Society of Breast Cancer Specialists (EUSOMA). Lancet Oncol 2012;13:e148-60.

11. Shachar SS, Hurria A, Muss HB. Breast Cancer in Women Older Than 80 Years. J Oncol Pract 2016;12:123-32. 
Jin Kyung An et al.

12. Poleszczuk J, Luddy K, Chen L, Lee JK, Harrison LB, Czerniecki $\mathrm{BJ}$, et al. Neoadjuvant radiotherapy of early-stage breast cancer and long-term disease-free survival. Breast Cancer Res 2017;19:75. 